\title{
The Exact Foldy-Wouthuysen Transformation for a Dirac Theory with the complete set of CPT-Lorentz violating terms
}

\author{
Bruno Gonçalveș 1 , Mário M. Dias Júnior² and Baltazar J. Ribeird 3 \\ ${ }^{1,2}$ Instituto Federal de Educação, Ciência e Tecnologia Sudeste de Minas Gerais \\ IF Sudeste MG, 36080-001, Juiz de Fora - MG, Brazil \\ ${ }^{3}$ Centro Federal de Educação Tecnológica \\ CEFET, 37.250-000, Nepomuceno - MG, Brazil
}

\begin{abstract}
The exact Foldy-Wouthuysen transformation is performed in order to study the Dirac field interacting with many possible external fields associated with CPTLorentz violation. We also derived the calculation of equations of motion as well as the generalized Lorentz force corrected by the mentioned external fields. The main point is the interaction between the Dirac particle and the terms that have the multiplication of electromagnetic field and the terms that break CPT-Lorentz. Finally, with the transformed Hamiltonian we were able to write an expression for the bound state of the theory and analyze it in the atomic experiments context. This result is an analytical expression that gives the possibility of the weakness of CPT/Lorentz terms to be compensated by the presence of a strong magnetic field.
\end{abstract}

Keywords: Dirac equation, CPT and Lorentz violating terms, Exact FoldyWouthuysen transformation.

PACS: 03.65.Pm; 11.30.Er

\section{Introduction}

The study of the possibility of the violation of CPT-Lorentz symmetries has received significant attention of the scientific community in the last years. For example, the CPT-Lorentz symmetries violation caused by terms in the action of quantum fields was considered in [1]. There are also great efforts about studding weakly broken CPT-Lorentz symmetries not only in the mathematical point of view. In Ref. [2], it is possible to see that some physical consequences may arise from the assumption that these symmetries can be in fact introduced into the action of the theory. In this context, this subject becomes important not only for theoretical physics. There is a growing expectation about experimental evidences of such violation. In Ref. 3] one can find an overview of all the possibilities of breaking CPT-Lorentz symmetries. There are many other recent Refs. ([4, 5]) that treat the possibility of CPT-Lorentz symmetry breaking in a more phenomenological point of view. But, in fact, the main part of the study of scientific

\footnotetext{
${ }^{1}$ E-mail: bruno.goncalves@ifsudestemg.edu.br

${ }^{2}$ E-mail: mardiasjr@yahoo.com.br

${ }^{3}$ E-mail: baltazarjonas@nepomuceno.cefetmg.br
} 
community remains on the search for the correct theory that could give the right interpretation for this symmetry breaking.

It is possible to find many references in the literature that support the evidence of this form of symmetry breaking. The parameters for experimental verification scenario are completely related to the consistency at the quantum level of the theory. For this reason the renormalization structure of theories with broken symmetries is an important feature to be analyzed (see, e.g., Ref. [6] for a brief and qualitative review of the renormalization properties of quantum field theories in curved spacetime in the presence of CPT-Lorentz violating terms).

A possible connection between theories with CPT-Lorentz violating terms and Cosmology (for baryogenesis considerations see [7]) worth to be analyzed, especially in the remarkable scenario of cosmic microwave radiation. In Ref. [8] there is an overview about the possibility of the Lorentz symmetry breaking manifestation. A possible candidate to produce the violation is the torsion field (except for unitarity problem). It is very interesting to see [9, 10] that this field could be generated from the symmetry breaking. These papers are showing the connection between the mathematical symmetry violation and some physical theory. It is also known that the torsion field, if it really exists, is very weak. But the main point here, is to achieve an interpretation to each of the CPT-Lorentz terms in such a way that it would be possible to search for some experimental result to support these theoretical results. The interaction between the spacetime torsion and the spinor field (and the spinning particle) is described in [11, 12, 13, 14]. In fact, not only torsion, but all the appropriated CPT-Lorentz violating terms would be one of the possible candidates to play the role to describe the known anisotropy related to cosmological perturbations. Another example is shown in [15] were the photon contribution to the divergences and conformal anomaly in the theory with CPT-Lorentz violating terms has been evaluated.

However, if we focus our attention on the torsion field (that has direct connection to CPTLorentz violation), in order to obtain a better interpretation about physical properties of the Dirac equation, it is important to consider the nonrelativistic approximation of the theory. In the Refs. 16, 17, 18, the Foldy-Wouthuysen transformation (FWT) has been obtained for the fermion field coupled to the combined electromagnetic and torsion fields. The FW method is a direct calculation that allows to extract physical information of the Dirac action (see e.g. [19, 20]). It is one of the methods to extract physical information from the Dirac Hamiltonian that is not diagonal from the beginning. That is the case when the CPT-Lorentz terms are taken into account.

Furthermore, it is possible to study the cases when not only the torsion field is considered. We can now think of which would be the appropriate approach to get physical information from a Hamiltonian that contains the other different terms that break CPT-Lorentz. The first point would be to choose the correct Hamiltonian, once none of the terms is experimentally verified. It can not be constructed using some empirical insights. But, the question here is a little bit more complicated. After the analytical Hamiltonian is presented, performing the FWT is not straightforward. The calculations may become cumbersome and, in the practical point of view, the analysis of the result could be lost into a very complex expression for the transformed equation. Then, this method, that was used before, when the unique term of interest was the torsion, seems to not work when the intention is to perform the calculus for more CPT-Lorentz 
breaking terms.

In this paper, we discuss the method to derive the physical analysis from the initial Hamiltonian. The EFWT is the more economic (in the algebra) and the more reliable approach to give the information in which we are interested. Another advantage that this transformation has is the fact that it allows us to use some specific notations that make all the steps, during the calculations, very clear. Then, in the end, the transformed equations have results that can be easily compared to the ones known from the literature.

In Ref. [21], there is an algorithm that shows how to construct a general Hamiltonian describing the 80 new cases of CPT-Lorentz violating terms in the Dirac equation that can be analyzed with the EFWT. The focus of the present work is precisely the development of EFWT, considering the whole situation presented in the last related work as well as the calculation of equations of motion. So, here we are going to treat the torsion as a particular case of the equations presented here, as well as each other terms in the complete Hamiltonian. Our aim is to get the most general result that can be written to perform the physical analysis for the CPT-Lorentz violating terms using the method we believe that is the most complete one to perform this kind of study, that is the EFWT. We also explore the possibility of combining such equations in order to obtain an expression to describe the dynamic of the particle.

Although FWT 22] provides, in general, more detailed information about the nonrelativistic approximation, there is a considerable advantage in the construction of the EFWT [23, 24, 25, 26, 27, 28. Using the Exact transformation, the risk of missing some important terms is lower 4. However, the possibility of performing exact transformation depends on the existence of the so called involution operator on the external classical fields and, from the mathematical point of view, EFWT is more complex [24, 25].

The paper is organized as follows. In the next section we present a brief summarize about EFWT. Coming up we consider the 80 cases mentioned above of CPT-Lorentz violating terms in the Dirac equation and perform EFWT. After that, the equations of motion are presented as a section and a more general Lorentz force is obtained. In the section 5 we present an equation for the bound state of this theory. Such equation is analyzed in the context of the CPT-Lorentz atomic experiments. In section 6 we draw our conclusions. Throughout the paper we use Greek letters for the indices which run from 0 to 3 . Latin indices are used for the space coordinates and run from 1 to 3 .

\section{Exact Foldy-Wouthuysen transformation}

We present in this section a brief review about EFWT [27]. We begin with the representation of even and odd operators in the FW context

$$
M_{(E V E N)}=\frac{1}{2}(M+\beta M \beta) \quad \text { and } \quad M_{(O D D)}=\frac{1}{2}(M-\beta M \beta) .
$$

The spinor transforms according to the following equations

$$
\psi^{t r}=U \psi, \quad \psi=U^{*} \psi^{t r},
$$

\footnotetext{
${ }^{4}$ See [27] for the spinor field in the weak gravitational field case.
} 
where $U$ is an unitary operator. After leading the last equation to the relation $i \partial \psi / \partial t=H \psi$ we have

$$
\mathcal{H}^{t r}=U \mathcal{H} U^{*}-i U \dot{U}^{*}
$$

where we have considered $i \partial \psi^{t r} / \partial t=\mathcal{H}^{t r} \psi^{t r}$. We focus on time independent external fields, then the last term in the equation (3) is zero. After considering the commutation relation $\left[\beta, \mathcal{H}^{t r}\right]=0$, one can show that $\left[U^{*} \beta U, \mathcal{H}\right]=0$ and a possible choice to play the hole of the quantity $U^{*} \beta U$ is

$$
U^{*} \beta U=\frac{\mathcal{H}}{\sqrt{\mathcal{H}^{2}}}=\lambda
$$

In this case $\sqrt{\mathcal{H}^{2}}$ must be understood as a notation. The calculation of $\mathcal{H}^{2}$ is performed in the coordinate representation and as a next step it is necessary to write $\mathcal{H}^{2}$ in the momentum representation in order to derive its squared root. In the last equation, the quantity $\lambda$ is a Hermitian and unitary operator $\lambda^{2}=\lambda^{\dagger} \lambda=1$. We assume that the Hamiltonian is well defined and it does not possess zero eigenvalues [24, 25]. It is remarkable to say that to perform EFWT, the Hamiltonian must obey the following relation

$$
J \mathcal{H}+\mathcal{H} J=0
$$

where

$$
J=i \gamma^{5} \beta
$$

is Hermitian and unitary. It is important to note that this explicit form of involution operator is the one used in 21] to establish the criteria to perform the EFWT for CPT-Lorentz terms. Another useful information about $J$ is the relation $J \beta+\beta J=0$. The EFWT is performed if we consider the relation $U=U_{2} U_{1}$ where

$$
U_{1}=\frac{1}{\sqrt{2}}(1+J \lambda) \quad \text { and } \quad U_{2}=\frac{1}{\sqrt{2}}(1+\beta J) .
$$

Consequently

$$
U_{1} \lambda U_{1}^{\dagger}=J \quad \text { and } \quad U_{2} \lambda U_{2}^{\dagger}=\beta .
$$

It is not difficult to check that the equations (7) satisfy the relation $U \lambda U^{\dagger}=\beta$. From the equation (4) it is easy to see that

$$
\beta=U \lambda U^{*}=\frac{\mathcal{H}^{t r}}{\sqrt{\left(\mathcal{H}^{t r}\right)^{2}}}
$$

and the quantity $\mathcal{H}^{t r}$ is given by $\mathcal{H}^{t r}=\beta \sqrt{\left(\mathcal{H}^{t r}\right)^{2}}$ which is an even Hamiltonian. Now and so on we denote the terms with "tr" index as the transformed ones and such terms belong to the final transformed Hamiltonian.

In the practical sense, performing all the steps to get $\mathcal{H}^{t r}$ is necessary to know the operator $U$ as a solution of the equation (4). Let us consider $U=\sqrt{\beta \lambda}$ as a solution. The operator $U$ has the property $U \beta=U^{*} \beta$. Now, from equation (5) we have $J \sqrt{\mathcal{H}^{2}}=\sqrt{\mathcal{H}^{2}} J$ and $J \lambda+\lambda J=0$. These last relations imply that $U_{1} \mathcal{H} U_{1}^{\dagger}=J \sqrt{\mathcal{H}^{2}}$ and we finally have the transformed Hamiltonian

$$
\mathcal{H}^{t r}=U \mathcal{H} U^{*}=\beta\left[\sqrt{\mathcal{H}^{2}}\right]_{(E V E N)}+J\left[\sqrt{\mathcal{H}^{2}}\right]_{(O D D)},
$$


where even and odd operators are defined in equation (11). The quantities $\sqrt{\mathcal{H}^{2}}(E V E N)$ and $\sqrt{\mathcal{H}^{2}}(O D D)$ represent the even and odd squared Hamiltonian respectively. If $\left[\mathcal{H}^{2}, \beta\right]=0$ we have an even term, and if $\left\{\mathcal{H}^{2}, \beta\right\}=0$ an odd one. The equation (10) represents the EFWT. As a second step one should calculate $\mathcal{H}^{2}$ with the even and odd correct identification and then multiply them by either $\beta$ or $J$. The operation $\sqrt{\mathcal{H}^{2}}$ can be considered in different approaches. A simpler option is to write $\mathcal{H}=\mathcal{H}_{0}+\mathcal{H}_{\text {int }}$. The coupling constants of the interaction terms are present in $\mathcal{H}_{\text {int }}$. That is, in fact, the main difference between the usual FWT and the EFWT. The freedom in the choice of the expansion parameter may give us results with more familiar analytical form for the final expressions. This fact, in general, makes the physical analysis easier to perform. The free Hamiltonian is denoted by $\mathcal{H}_{0}$ and the interaction one by $\mathcal{H}_{\text {int }}$. Both terms in the equation (10) are even terms and for this reason the quantity $\mathcal{H}^{\text {tr }}$ does not mix upper spinor components with lower ones.

\section{Exact transformation with CPT}

We shall deal with the action of a Dirac fermion theory, including CPT-Lorentz symmetry breaking terms

$$
S=\int d^{4} x \sqrt{-g}\left\{\frac{i}{2} \bar{\Psi} \Gamma^{\mu} D_{\mu} \Psi-\frac{i}{2} D_{\mu}^{\star} \bar{\Psi} \Gamma^{\mu} \Psi-\bar{\Psi} M \Psi\right\}
$$

The CPT-Lorentz symmetry breaking terms can be written in the following way 3 ]

$$
D_{\mu}=\nabla_{\mu}-i e A_{\mu} ; \quad D_{\mu}^{\star}=\nabla_{\mu}+i e A_{\mu} ; \quad \Gamma^{\mu}=\gamma^{\mu}+\Gamma_{1}^{\mu} ; \quad M=m+M_{1} .
$$

The quantities $\Gamma_{1}^{\nu}$ and $M_{1}$ can be written in terms of the CPT-Lorentz violating parameter 5 $a_{\mu}, b_{\mu}, m_{5}, c^{\mu \nu}, f^{\mu}, e^{\mu}, g^{\mu \nu \lambda}, H_{\mu \nu}$, in the following way

$$
\Gamma_{1}^{\nu}=c^{\mu \nu} \gamma_{\mu}+d^{\mu \nu} \gamma_{5} \gamma_{\mu}+e^{\nu}+i f^{\nu} \gamma_{5}+\frac{1}{2} g^{\lambda \mu \nu} \sigma_{\lambda \mu}
$$

and

$$
M_{1}=a_{\mu} \gamma^{\mu}+b_{\mu} \gamma^{5} \gamma^{\mu}+i m_{5} \gamma^{5}+\frac{1}{2} H^{\mu \nu} \sigma^{\mu \nu}
$$

We adopt notations as described in 31] for Dirac Matrices and the useful notations for $P_{i}$, used in [21]

$$
P_{\nu}^{0}=\left(0, P_{i}\right), \quad \bar{P}_{\nu}=P_{\nu}^{0}-e A_{\nu} \quad \text { and } \quad P_{\nu}^{*}=\bar{P}_{\nu}-\frac{i}{2} \nabla_{\nu} .
$$

Under such definitions, equation (111) takes the form

$$
S=\int d^{4} x \bar{\Psi}\left\{i \Gamma^{\mu} D_{\mu}+\frac{i}{2}\left(\nabla_{\mu} \Gamma^{\mu}\right)-M\right\}
$$

After such considerations, taking the equations of motion for $\psi$ in the Schrodinger form $i \partial_{t} \psi=\mathcal{H} \psi$, one can check that

$$
i \nabla_{0} \psi=\left\{\gamma_{0}-\gamma_{0}\left(c^{\mu 0} \gamma_{\mu}+d^{\mu 0} \gamma_{5} \gamma_{\mu}+e^{0}+i f^{0} \gamma_{5}+\frac{1}{2} g^{\lambda \mu 0} \sigma_{\lambda \mu}\right) \gamma_{0}\right\} \times\left[M+\left(P_{\nu}^{*} \Gamma^{\nu}\right)\right] \Psi .
$$

\footnotetext{
${ }^{5}$ The authors discuss in ref. [29, 30] some aspects about origin of these terms.
} 
In order to perform the EFWT, it is necessary that the Hamiltonian admits the involution operator [23, 24, 25, 27]. The most general form of the equation (17) that admits the involution operator is presented in Ref. [21. In such work the authors present a complete table which contains the 80 cases of CPT-Lorentz violating terms in the modified Dirac equation that admits EFWT. For the sake of completeness, we consider in the present work the complete Hamiltonian with CPT-Lorentz breaking terms of Dirac Theories admitting EFWT

$$
\begin{aligned}
\mathcal{H} & =m\left(\gamma^{0}-\gamma^{0} c_{00}+i \gamma^{5} f_{0}+\gamma^{i} \gamma^{5} d_{i 0}+\frac{i}{2} \alpha^{i} g_{i 00}\right)+\bar{P}_{l}\left(\gamma^{0} e^{l}+\alpha_{i} c^{i l}-\gamma^{5} d^{0 l}+\alpha^{l}\right. \\
& \left.+\frac{1}{2} \gamma^{0} \sigma^{i j} g_{i j l}-\alpha^{l} c^{00}+i \gamma^{5} \gamma^{l} f_{0}+\gamma^{i} \gamma^{5} \gamma^{l} d_{i 0}+\frac{i}{2} \alpha^{i} \gamma^{l} g_{i 00}\right) \\
& +\alpha^{l} a_{l}-\gamma^{5} b_{0}+\frac{1}{2} \gamma^{0} \sigma^{i j} H_{i j} .
\end{aligned}
$$

Interaction terms of second order are very small and for this reason they can be neglected in this last equation and so on. We calculate the squared Hamiltonian as a first step to get exact transformation. We adopt the quantity $\overline{\mathcal{H}}^{2}$ to represent the squared Hamiltonian. It is convenient to write the quantity $\overline{\mathcal{H}}^{2}$ in the form

$$
\overline{\mathcal{H}}^{2}=(1+\bar{A})\left[\left(\delta_{i j}+B_{i j}\right) \bar{P}^{i}+\bar{C}_{j}\right]^{2}+\bar{D}+m^{2},
$$

where we define the following notations

$$
\begin{aligned}
\bar{A} & =-4 d_{i 0} \gamma^{5} \alpha^{i}-4 i f_{0} \gamma^{0} \gamma^{5}+i g_{i 00} \gamma^{0} \alpha^{i}, \\
\bar{B}_{i j} & =\frac{1}{2}\left[-2 c_{i j}-2 \gamma^{5} \alpha^{i} d_{0 j}-2 \gamma^{5} \alpha^{i} d_{j 0}-2 \gamma^{0} \gamma^{5} \epsilon^{l m i} g_{l m j}+2 i \gamma^{0} \gamma^{5} g_{t i j} \Sigma^{t}+\frac{i}{3} g_{i 00} \gamma^{0} \alpha_{j}\right] \\
\bar{C}_{j} & =\frac{1}{2}\left[2 m e_{j}+m \sigma^{l m} g_{l m j}-2 i m g_{j 00}-4 m \gamma^{0} \gamma^{5} d_{j 0}-2 a_{j}-2 \gamma^{5} \alpha^{j} b_{0}-2 H_{l m} \epsilon^{l m j} \gamma^{0} \gamma^{5}\right. \\
& \left.-m g_{l 00} \epsilon^{l j k} \Sigma_{k}-2 i m \gamma^{0} \gamma^{5} d_{m 0} \epsilon^{j m k} \Sigma_{k}+2 i \gamma^{0} \gamma^{5} H_{m j} \Sigma^{m}\right], \\
\bar{D} & =-2 m^{2} c_{00}-2 m^{2} \gamma^{5} \alpha^{i} d_{i 0}+m \sigma^{i j} H_{i j} \\
& +\left(1-2 i d_{i 0} \gamma^{5} \alpha^{i}-2 i f_{0} \gamma^{0} \gamma^{5}+\frac{i}{3} g_{i 00} \gamma^{5} \alpha^{i}\right) \frac{i \hbar e}{m c} \Sigma_{k} B^{k} .
\end{aligned}
$$

These notations are used to simplify the algebra and make the interpretation of the results easier to perform. In this form, it is possible, for example, to direct identify which terms are linked with the kinetic part of the Hamiltonian.

In accordance to EFWT transformation, we should take squared root of even terms in equation (25) and multiply them by $\beta$. On the other hand, the squared root of odd terms should be multiplied by $J=i \gamma^{5} \beta$. Moreover, such procedure is not quite trivial in the situation when there are many odd terms. We present a new point of view to perform the exact transformation. Note that it is possible to show that the equation (10) is completely equivalent to

$$
\mathcal{H}^{t r}=J \frac{1}{2}\left(\sqrt{\mathcal{H}^{2}}-\beta \sqrt{\mathcal{H}^{2}} \beta\right)+\beta \frac{1}{2}\left(\sqrt{\mathcal{H}^{2}}+\beta \sqrt{\mathcal{H}^{2}} \beta\right) .
$$

This last relation allows us to perform the EFWT where many odd terms are present. According to equation (21), it is possible to take as a first step, the squared root of the complete Hamiltonian and as a second step, identify and separate even and odd terms. 
In order to take the squared root we shall consider that $m^{2} \gg \overline{\mathcal{H}}^{2}$, in the equation (19), so well as the expansion. These considerations allow us to assert that the following equation

$$
\mathcal{H}^{2}=m^{2}\left(1+\frac{\overline{\mathcal{H}}^{2}}{m^{2}}\right)
$$

can be written as

$$
\sqrt{\mathcal{H}^{2}}=m\left(1+\frac{\overline{\mathcal{H}}^{2}}{2 m^{2}}\right)
$$

where $\overline{\mathcal{H}}^{2}$ is given by relation (19). After some algebra we have the following equation for the transformed Hamiltonian

$$
\mathcal{H}^{t r}=\beta m+\frac{1}{2 m}\left(1+A^{t r}\right)\left[\left(\delta_{i j}+B_{i j}^{t r}\right) \bar{P}^{i}+C_{j}^{t r}\right]^{2}+D^{t r},
$$

where

$$
\begin{aligned}
A^{t r} & =-4 \beta \Sigma^{i} d_{i 0}+4 f_{0}+\Sigma^{i} g_{i 00}, \\
\bar{B}_{i j}^{t r} & =\frac{1}{2}\left[-2 \beta c_{i j}+2 \beta \Sigma^{i} d_{0 j}+2 \beta \Sigma^{i} d_{j 0}-2 i \epsilon^{l m i} g_{l m j}-2 g_{t i j} \Sigma^{t}+\frac{1}{3} g_{i 00} \Sigma_{j}\right], \\
\bar{C}_{j}^{t r} & =\frac{1}{2}\left[2 m \beta e_{j}+m \beta g_{l m j} \epsilon^{l m k} \Sigma_{k}-4 i m d_{j 0}-2 \beta a_{j}+2 \beta \Sigma^{j} b_{0}-2 i \epsilon^{l m j} H_{l m}\right. \\
& \left.-m \beta \epsilon^{l j k} \Sigma_{k} g_{l 00}+2 m \epsilon^{j m k} \Sigma_{k} d_{m 0}-2 i m \beta g_{j 00}-2 \Sigma^{m} H_{m j}\right], \\
\bar{D}^{t r} & =-2 m^{2} \beta c_{00}+2 m^{2} \beta \Sigma^{i} d_{i 0}+m \beta \epsilon^{i j k} \Sigma_{k} H_{i j} \\
& +\left[\beta\left(1+2 i \Sigma^{i} d_{i 0}\right)+2 f_{0}+\frac{1}{3} \Sigma^{i} g_{i 00}\right] \frac{i \hbar e}{m c} \Sigma_{k} B^{k} .
\end{aligned}
$$

The equation (24) presents a well known structure. According to this equation, the first term corresponds to rest energy. The second one represents the kinetic term. It is a term of the kind $\mathbf{P}-e \mathbf{A}$ and one can imagine the quantity $C_{j}^{t r}$ (in the situation where $B_{i j}^{t r}=0$ ), as being an analogous term of a gauge transformation to $\bar{P}^{i}$. The quantity $1+A^{t r}$ can be seen as a correction to the general form of the kinetic energy. The last term in the equation (24) corresponds to a pure external interaction.

\section{Equations of motion}

We perform in this section the calculations of equations of motion. We also explore the possibility of combining such equations in order to obtain an expression to describe the dynamic of the particle as a result. The Lorentz force corrected by CPT-Lorentz violating terms is obtained. Let us begin by taking into account the two components spinor

$$
\psi=\left(\begin{array}{l}
\phi \\
\chi
\end{array}\right) \exp ^{-i m t}
$$

and write the Dirac equation in the Schrödinger form $i \partial_{t} \psi=\mathcal{H} \psi$. After some algebra, one can get the following Hamiltonian to $\phi$

$$
\mathcal{H}=\frac{1}{2 m}\left\{(1+A)\left[\left(\delta_{i j}+B_{i j}\right) \bar{P}^{i}+C_{j}\right]^{2}+D\right\}
$$


where

$$
\begin{aligned}
A & =-4 \sigma^{i} d_{i 0}+4 f_{0}+\sigma^{i} g_{i 00}, \\
B_{i j} & =-c_{i j}+\sigma^{i} d_{0 j}+\sigma^{i} d_{j 0}-i \epsilon^{l m i} g_{l m j}-g_{t i j} \sigma^{t}+\frac{1}{3} g_{i 00} \sigma_{j}, \\
C_{j} & =m e_{j}+\frac{1}{2} m g_{l m j} \epsilon^{l m k} \sigma_{k}-2 i m d_{j 0}-a_{j}+\sigma^{j} b_{0}-i \epsilon^{l m j} H_{l m} \\
& -\frac{1}{2} m \epsilon^{l j k} \sigma_{k} g_{l 00}+m \epsilon^{j m k} \sigma_{k} d_{m 0}-i m g_{j 00}-\sigma^{m} H_{m j}, \\
D & =-2 m^{2} c_{00}+2 m^{2} \sigma^{i} d_{i 0}+m \epsilon^{i j k} \sigma_{k} H_{i j} \\
& +\left[1+2 i \sigma^{i} d_{i 0}+2 f_{0}+\frac{1}{3} \sigma^{i} g_{i 00}\right] \frac{i \hbar e}{m c} \sigma_{k} B^{k} .
\end{aligned}
$$

In order to quantize Hamiltonian (27) and to write semi-classical equations of motion 6 let us consider the following relations

$$
i \hbar \frac{d \hat{x}_{i}}{d t}=\left[\hat{x}_{i}, \mathcal{H}\right], \quad i \hbar \frac{d \hat{p}_{i}}{d t}=\left[\hat{p}_{i}, \mathcal{H}\right] \quad \text { and } \quad i \hbar \frac{d \hat{\sigma}_{i}}{d t}=\left[\hat{\sigma}_{i}, \mathcal{H}\right] .
$$

So we get

$$
\begin{gathered}
\frac{d \hat{x}_{i}}{d t}=\frac{1}{m}(1+A)\left[\left(\delta_{i j}+2 B_{[i j]}\right) \bar{P}^{j}+C_{i}\right] \\
\frac{d \hat{p}_{i}}{d t}=\frac{1}{2 m}\left\{-\frac{\partial A}{\partial x_{i}}\left[\left(\delta_{k j}+B_{k j}\right) \bar{P}^{k}+C_{j}\right]^{2}\right. \\
-(1+A)\left[2\left(\frac{\partial}{\partial x_{i}}\left[\left(\delta^{l j}+B^{l j}\right) \bar{P}_{l}+C_{j}\right]\right)\left[\left(\delta_{k j}+B_{k j}\right) \bar{P}^{k}+C_{j}\right]-\frac{\partial D}{\partial x^{i}}\right\} \\
=e v_{j} \frac{\partial A^{j}}{\partial x_{i}}
\end{gathered}
$$

and

$$
i \hbar \frac{d \hat{\sigma}_{i}}{d t}=\varepsilon_{i j k} R_{j} \sigma_{k}+C_{i j} \sigma^{j}
$$

where

$$
\begin{aligned}
R_{j} & =\left(-4 i m d_{j 0}+i m g_{j 00}\right) v^{2}+\left(2 i m d_{0 l} \delta_{j m}+2 i m d_{l 0} \delta_{j m}-2 i m g_{m l j}+\frac{2}{3} i m g_{l 00} \delta_{j m}\right) v_{l} v_{m} \\
& +\left(2 i b_{0} \delta_{i j}+2 i H_{i j}\right) v^{i}+2 i m d_{j 0}-\frac{\hbar e}{m^{2} c}\left(1+2 f_{0}\right) B_{j}
\end{aligned}
$$

and

$$
C_{i j}=\left(2 i m g_{k i j}+i m g_{i 00} \delta_{j k}+2 i m d_{i 0} \delta_{j k}-i m g_{k 00} \delta_{i j}-2 i m d_{k 0} \delta_{i j}\right) v^{k}+2 i H_{i j} .
$$

Taking the derivative of equation (30) with respect to time one can get the generalized Lorentz force

$$
m \frac{d v_{i}}{d t}=\frac{d \lambda_{i j}}{d t} m v^{j}+\frac{d C_{i}}{d t}+\left(\delta_{i j}+\lambda_{i j}\right)[-e \vec{E}+e \vec{v} \times \vec{B}]^{j}
$$

where

$$
\lambda_{i j}=A \delta_{i j}+2 B_{[i j]}
$$

\footnotetext{
${ }^{6}$ After the calculus we make $\hbar \rightarrow 0$ in the same procedure adopted in 32 .
} 
and the quantities $A$ and $B_{i j}$ are described by equations (28). The equation (35) represents the Lorentz force corrected by CPT-Lorentz violating terms. It is worth noting that if the quantities $c_{00}, d_{i 0}, c_{i j}, g_{l j i}$ and $g_{i 00}$ are null, one can get the Lorentz force as a particular case of equation (35). The first term in such equation represents a dragging term once it is proportional to the velocity. The second one is related to an external force and the last one is a correction to the the very well known quantity $e(\mathbf{E}+\mathbf{v} \times \mathbf{B})$.

\section{CPT tests, a perspective}

We present in this section a brief perspective about experimental tests, tanking into account the EFWT whole scenario, including the 80 possible cases of CPT-Lorentz violation in the Dirac equation. As previously explained, we deal with the Hamiltonian of interaction between the Dirac spinor and external fields.

A consistent theoretical framework about CPT-Lorentz breaking tests (in particle and atomic systems) can be found in [33, 34, 35. Such framework incorporates CPT-Lorentz violation by using the so called standard model extension (SME) [35] and the search for new signatures is possible. Nevertheless, this subject is very much extensive and a deep approach here would not be compatible with the context of this work.

It is well known that Quantum Electrodynamics systems are extremely proliferous in the scope of CPT-Lorentz violation tests, since it is sensitive to very low energies. One can name some examples of experiments involving atomic physics experiments as Penning-Trap, ClockComparison, Torsion Pendulum, Hydrogen-Antihydrogen Experiments, Spin-Polarized Matter, Muon Experiments, among others (see refs. [36, 37, 38, 39, 40, and references cited there in.). Each one of the experiments mentioned present a very specific bound state and the magnitude of such bounds makes possible to determine which kind of experiments should be performed [41.

A very natural question arises here. Is there a bound state associated to the EFWT for a Dirac theory related to the 80 new CPT-Lorentz violating terms? In order to answer this question, let us take into account the Lorentz violating potential $V$, which obeys the following relation 37 ]

$$
V=-\tilde{b}_{j} \sigma_{j}
$$

where $\sigma$ represents the spin matrices. After some algebra, one can write the corresponding bound in the following way 7

$$
\tilde{b}_{j}=b_{j}-\frac{1}{2} \epsilon^{j l m} H_{l m}-m d_{j 0}-\left[1+2 i \sigma^{i} d_{i 0}+2 f_{0}+\frac{1}{3} \sigma^{i} g_{i 00}\right] \frac{i \hbar e}{2 m^{2} c} B_{j} .
$$

The bound presented in the last equation enables us to consider the possibility about getting an indication of possible atomic experiments [41. In fact, the magnitude of the magnetic field plays a crucial role in the determination of such experiments. However, direct analysis of the equation (38) to predict which is the most appropriate experiment is not straightforward.

\footnotetext{
${ }^{7}$ Note that the Lorentz violating potential comes, naturally, from the equation (28).
} 
As an example of this procedure, we consider the following Hamiltonian obtained after FoldyWouthuysen transformation

$$
\mathcal{H}=m+\frac{p^{2}}{2 m}+a_{0}-m c_{00}+\left(-b_{j}+m d_{j 0}+\frac{1}{2} \epsilon_{j k l} H_{k l}\right) \sigma^{j}+\left[-a_{j}+m\left(c_{0 j}+c_{j 0}\right)\right] \frac{p_{j}}{m},
$$

where all terms in the last equation where previously defined in section (3). This Hamiltonian presents the bound

$$
\tilde{b}_{j}=b_{j}-\frac{1}{2} \epsilon_{j k l} H_{k l}-m d_{j 0} .
$$

Such bound is compatible with torsion pendulum experiments [43]. Clearly, the result presented in the equation (38) is more general than the one presented in the equation (40). If one considers the situation where the magnetic field is null, it is possible to get the bound state of the torsion pendulum experiment, described by (40) as a particular result. We also observe that the Hamiltonian described by the equation (39) can be seen as a particular case of the equation (27).

If we consider the same approach, that is, consider the only non-vanishing interaction terms to be $a_{0}, c_{00}, b_{j}, d_{j 0}, H_{k l}$ and $c_{o j}$, from the equation (27), after some algebra, it is possible to obtain the same structure as the one observed on the equation (39). The terms $a_{o}$ and $b_{j}$ will not be present since they were not taken into account in initial Hamiltonian. The reason for this was the non acceptance of these terms to the EFWT criteria. Nevertheless, all the other quantities are present and have a more general form. In this sense it is possible to consider the equation (27) as a more complete case, despite the torsion field $\left(b_{j}\right)$ and $a_{j}$ are not present.

Another result that should be analyzed comes from the equation (35). This equation is related to the possibility of understanding the real particle behavior due to the interactions with the external fields. As an example, let us cite the interactions mixing terms between magnetic field $\mathbf{B}$ and the CPT-Lorentz terms of the kind $\lambda_{i j} B$.

It is possible to imagine the case where the modulus of $\mathbf{B}$ is sufficiently high in order to compensate the weakness of the interactions. Possibility to measure such quantities in an indirect way (for example, using atomic and molecular physics) is contemplated if one considers the situation where a gas of electrons is present. In principle, it is possible to make a prediction of the motion generated by these new terms.

\section{Conclusions and discussions}

The EFWT was here considered and performed in the context of all the 80 CPT-Lorentz violating terms, in the Dirac equation. The first result of the work is given by the equation (24), which presents a well known structure and it is possible to identify in it terms like the rest, the kinetic and the potential energy. All of them corrected by the CPT-Lorentz violating terms.

It is worth noting that EFWT is not quite trivial, specifically in the presence of many odd terms. Nevertheless, the equation (21) introduces a new way of performing the EFWT, such that it becomes easier to consider, in the situation when many odd terms are present. For this reason, the calculation procedure adopted here can be used as a guidance to the kind of situation described above. 
Other results of the work are the semi-classical equations of motion for $\hat{x}_{i}, \hat{p}_{i}$ and $\hat{\sigma}_{i}$, given respectively by the equations (30), (31) and (32). We have shown that it is possible to combine equations of motion to get a generalized Lorentz force corrected by CPT-Lorentz violating terms, given by the equation (35). Such equation presents dragging term, external force and a factor of the kind $\delta^{i j}+\lambda^{i j}$ correcting the quantity so much well known $e(\mathbf{E}+\mathbf{v} \times \mathbf{B})$. The quantity $\lambda^{i j}$ is given by the relation (36). As expected, one can get the usual Lorentz force by taking the limit in the absence of external fields in the equation (35).

In the last section we have highlighted that the bound state equation is important in order to get a better understanding about which kind of atomic experiments should be performed. In the context of the EFWT for a Dirac Theory with the complete set of CPT-Lorentz violating terms, we have presented the bound state for this theory, given by the equation (38). Such equation allow us to conclude that the kind of atomic experiments that should be performed in this case, depends on the magnitude of the magnetic field. We also observe that if magnetic field is null, in the equation (38), on can recover the well known bound state, given by the equation (40), and the atomic experiment associated would be the pendulum experiments.

Another result from the last section is the possibility of understanding the particle behavior in the presence of interactions with the external fields. One can imagine interactions mixing terms between magnetic field $B$ and the CPT-Lorentz terms of the kind $\lambda_{i j} B$. From the experimental point of view, it is an interesting situation when the modulus of $\mathbf{B}$ is sufficiently high in order to compensate the external fields effects.

In Ref. [44, for example, the idea of the possibility of detecting gravitational waves with atom interferometers is explained. In the present work we deal with an analog measuring problem, that is, the weakness of the external interaction terms. Our main result (35) shows that the such weakness may be compensated by the magnetic field. We intend to perform the derivation of the same equations for a gas of spin in a near future.

\section{Acknowledgments}

The authors wish to thank Prof. Ilya L. Shapiro for the initial discussions about the problem.

BG and MJ are grateful to Fundação Nacional de Desenvolvimento da Educação (FNDE) for financial support. Posthumously, BR and BG thank deeply Prof. Wilson Oliveira by advices and discussions, during their academic formation.

\section{References}

[1] Kostelecky, A. and Russell, N.; Rev. Mod. Phys. 83, 11 (2011); arXiv:0801.0287,

Kostelecky, A.V. and Tasson, J.D.; Phys.Rev. D83, 016013 (2011), arXiv:1006.4106.

[2] Kostelecky, V.A. and Russell, N.; Data Tables for Lorentz and CPT Violation, 2013 edition, arXiv:0801.0287v6.

[3] Colladay, D. and Kostelecky, A.; Phys.Rev. D55, 6760 (1997); Phys.Rev. D58, 116002 (1998).

[4] Scarpelli, A.P.B.; J. Phys. G: Nucl. Part. Phys. 39, 125001 (2012). 
[5] Casana, R.; Ferreira Jr, M.M.; Maluf, R.V. and Santos, F.E.P. dos; J. Physical Review D86, 125033 (2012).

[6] Shapiro, I.L.; CPT and Lorentz Symmetry: Proceedings of the Sixth Meeting, 184-187 (2014), arXiv:1309.4190v1.

[7] Bertolami, O.; Colladay, D.; Kostelecky, V.A.; and Potting, R.; Phys.Lett. B395 178, (1997), arXiv:hep-ph/9612437 v1.

[8] Russell, N.; Phys. Scr. 84, 038101 (2011), arXiv:1109.0768v1.

[9] Shapiro, I.L.; Phys. Repts. 357, 113 (2002), arXiv:hep-th/0103093v1.

[10] Kostelecky, V.A.; Phys. Rev. D69, 105009 (2004), arXiv:hep-th/0312310v2.

[11] Datta, B.K.; Nuovo Cim. 6B, 1-15; 16-28 (1971).

[12] Audretsch, J.; Phys.Rev. 24D, 1470 (1981).

[13] Hayashi, K.; Progr. Theor. Phys. 64, 866; 883 (1980).

[14] Rumpf, H.; Gen. Relat. Grav. 10, 509; 525; 647 (1979); 14, 773 (1982).

[15] Neto, T.P. and Shapiro, I.L.; Phys. Rev. D89, 104037 (2014), arXiv:1403.3152v1.

[16] Sabbata, V. de; Pronin, P.I. and Sivaram, C.; Int. J. Theor. Phys. 30, 1671 (1991).

[17] Bagrov, V.G.; Buchbinder, I.L. and Shapiro, I.L.; Izv. VUZov, Fisica (in Russian. (English translation: Sov.J.Phys.) 35, 5 (1992);

[18] Ryder, L.H. and Shapiro, I.L.; Phys. Lett. A245, 21-26 (1998), arXiv:hep-th/9805138v1.

[19] Jentschura, U.D. and Noble, J.H.; Phys. Rev. A47, 045402 (2014), arXiv:1312.3456v1.

[20] Obukhov, Y.N.; Silenko, A.J. and Teryaev, O.V.; Phys. Rev. D88, 084014 (2013), arXiv:1308.4552 1 .

[21] Gonçalves, B.; Obukhov, Y.N. and Shapiro, I.L.; Phys. Rev. D80, 125034 (2009), arXiv:0908.0437v1.

[22] Foldy, L.L. and Wouthuysen, S.; Phys. Rev. 58, 29 (1950).

[23] Case, K.M.; Phys. Rev. 95, 1323 (1954).

[24] Eriksen, E. and Kolsrud, M.; Nuovo Cim. Suppl. 18, 1 (1960).

[25] Nikitin, A.G.; J. Phys. A: Math. Gen. A31, 3297 (1998).

[26] Oliveira, C.G. de and Tiomno, J.; Nuovo Cim. 24, 672 (1962).

[27] Obukhov, Y.N.; Phys. Rev. Lett. 86, 192-195 (2001), arXiv:gr-qc/0012102v1. 
[28] Silenko, A.J. and Teryaev, O.V.; Phys. Rev. D71, 064016 (2005), arXiv:gr-qc/0407015v3; D76, 061101 (2007), arXiv:gr-qc/0612103v2; Obukhov, Y.N.; Silenko, A.J. and Teryaev, O.V.; Phys. Rev. D80, 064044 (2009), arXiv:0907.4367v2.

[29] Kostelecky, V.A. and Samuel, S.; Phys. Rev. D39, 683 (1989);

Kostelecky, A. and Potting, R.; Phys. Rev. D63, 046007 (2001), arXiv:hep-th/0008252v2.

[30] Jackiw, R. and Kostelecky, A.; Phys. Rev. Lett. 82, 3572-3575 (1999), arXiv:hep-ph/9901358v1.

[31] Bjorken, J.D. and Drell, S.D.; Relativistic Quantum Mechanics, (Mac-Graw Hill, San Francisco, 1964).

[32] Buchbinder, I.L. and Shapiro, I.L.; Phys. Lett. B151, 263-266 (1985).

[33] Kostelecky, V.A. and Samuel, S.; Phys. Rev. Lett. 63, 224 (1989); Phys. Rev. Lett. 66, 1811 (1991); Phys. Rev. D39, 683 (1989); Phys. Rev. D40, 1886 (1989).

[34] Kostelecky, V.A. and Potting, R.; Nucl. Phys. B359, 545 (1991); Phys. Lett. B381, 89-96 (1996), arXiv:hep-th/9605088v1; Kostelecky, V.A.; Perry, M. and Potting, R.; Phys. Rev. Lett. 84, 4541-4544 (2000), arXiv:hep-th/9912243v1.

[35] Colladay, D. and Kostelecky, V.A.; Phys. Rev. D55, 6760-6774 (1997), arXiv:hep-ph/9703464v1; Phys. Rev. D58, 116002 (1998), arXiv:hep-ph/9809521v1.

[36] Bluhm, R.; Kostelecky, V.A. and Russell, N.; Phys. Rev. Lett. 79, 1432-1435 (1997), arXiv:hep-ph/9707364v1; Phys. Rev. D57, 3932-3943 (1998), arXiv:hep-ph/9809543v1.

[37] Kostelecky, V.A. and Lane, C.D.; Phys. Rev. D60, 116010 (1999), arXiv:hep-ph/9908504v1.

[38] Bluhm, R.; Kostelecky, V.A. and Russell, N.; Phys. Rev. Lett. 82, 2254-2257 (1999), arXiv:hep-ph/9810269v1.

[39] Bluhm, R. and Kostelecky, V.A.; Phys. Rev. Lett. 84, 1381-1384 (2000), arXiv:hep-ph/9912542v1.

[40] Bluhm, R.; Kostelecky, V.A. and Lane, C.D.; Phys. Rev. Lett. 84, 1098-1101 (2000), arXiv:hep-ph/9912451v1.

[41] Kostelecky, A. and Russell, N.; Rev. Mod. Phys 83, 11 (2011), arXiv:0801.0287v7.

[42] Kostelecky, A. and Mewes, M.; Astrophys. J. Lett. 689, L1-L4 (2008), arXiv:0809.2846v2.

[43] Colladay, D.; AIP Conference Proceedings Vol. 1560, 137 (American Institute of Physics, St. Petersburg, 2012), arXiv:1208.3474v1.

[44] Tino, G.M. and Vetrano, F.; Class. Quantum Grav. 24, 2167 (2007). 